\title{
Study on Memory Function of Stroke Patients under Exercise Relearning Based on DWI Image Analysis Based on Optimized Registration Algorithm
}

\author{
Haihong Zhao $\mathbb{D},{ }^{1}$ Bo Li $\mathbb{D}^{2},{ }^{2}$ Xiaonian Zhang $\mathbb{D}^{3},{ }^{3}$ Xiaolin Guo $\mathbb{D}^{4},{ }^{4}$ and Lixia Qian $\mathbb{D}^{4}$ \\ ${ }^{1}$ Department of Neurorehabilitation, Shanxi Rongjun Hospital, Taiyuan 030000, Shanxi, China \\ ${ }^{2}$ Department of Rehabilitation Treatment, Shanxi Rongjun Hospital, Taiyuan 030000, Shanxi, China \\ ${ }^{3}$ Department of Neurorehabilitation, China Rehabilitation Research Center, Beijing 100068, China \\ ${ }^{4}$ Department of Imaging Medicine, Shanxi Bethune Hospital, Taiyuan 030000, Shanxi, China \\ Correspondence should be addressed to Lixia Qian; 13472101@smail.cczu.edu.cn
}

Received 19 June 2021; Accepted 11 August 2021; Published 22 December 2021

Academic Editor: Gustavo Ramirez

Copyright (C) 2021 Haihong Zhao et al. This is an open access article distributed under the Creative Commons Attribution License, which permits unrestricted use, distribution, and reproduction in any medium, provided the original work is properly cited.

\begin{abstract}
Objective. This paper uses an optimized registration algorithm to analyze the diffusion-weighted imaging (DWI) scan parameters of acute ischemic stroke (AIS) in the memory function of stroke patients under exercise relearning (MRP). Methods. This study used a random case-control study. 65 patients with stroke and hemiplegia were randomly divided into a control group: conventional rehabilitation intervention (32 cases), and a treatment group: MRP (33 cases). Each patient uses 4 parameters for DWI examination and obtains 4 sets of images, group 1 is the control sequence, group 2 uses parallel acquisition technology, group 3 uses parallel acquisition technology and reduces the number of excitations, group 4 uses parallel acquisition technology to reduce repetition time (TR) and echo time (TE) and enlarge the field of view, and the scan time of each group is $177,81,23$, and $18 \mathrm{~s}$ in sequence. At the time of enrollment and after 12 weeks of treatment, patients in each group were evaluated with Fugl-Meyer motor function score (FMA) and modified Pap index (MBI) for hand and wrist motor function and ADL. Results. After treatment, the FMA and MBI values of the treatment group were significantly higher than those of the control group $(P<0.05)$. Conclusion. By adopting a parallel acquisition technique and reducing the number of excitations (group 3) scanning scheme, not only the scanning time is significantly shortened, but also the image quality can meet the diagnostic requirements, which has great application value for AIS patients who need emergency treatment. MRP can obviously promote the hand and wrist motor function and daily living ability of stroke hemiplegic patients.
\end{abstract}

\section{Introduction}

Stroke (AIS) is a major disease that seriously threatens human health. Both mortality and disability rate are very high. Most survivors are left with different degrees of disability, generally with motor dysfunction, especially paralyzed hand and wrist movements. Dysfunction has a greater impact on patients' independence in daily life. The recovery of hand and wrist motor function in stroke paralysis has always been a problem, which has caused the decline of daily living ability to a large extent. Diffusion-weighted imaging (DWI) can detect cerebral infarction early, assess prognosis, and make a differential diagnosis of other diseases with similar clinical signs and signs to AIS, which plays an important role in the imaging diagnosis of ASI. However, conventional scanning parameters are not suitable for severe and uncooperative patients and cannot be widely used to screen the effects of thrombolytic therapy in ASI patients. Therefore, this study aims to explore the application value of DWI after optimization of scanning parameters to AIS patients and to study the effect of exercise relearning (MRP) training method on the comprehensive function of hands and wrists and the independence of daily life in hemiplegic patients and the role of hand and wrist motor function in stroke hemiplegia. On the premise of meeting the requirements of imaging diagnosis, the scan time should be 
shortened as much as possible, so as to be more effectively applied to the examination of AIS patients [1].

\section{Materials and Methods}

2.1. General Information. The thesis selected 65 patients with stroke and hemiplegia who were outpatient and inpatient in our hospital from December 2018 to December 2019. The patients were randomly divided into a treatment group and a control group, including 33 cases in the treatment group and 32 cases in the control group. The inclusion criteria are as follows: (1) the diagnosis meets the diagnostic criteria established by the 1995 National Fourth Cerebrovascular Disease Conference; (2) age is 12-82 years; (3) all cases are diagnosed clearly, vital signs are stable, and no more stroke symptoms for more than 48 hours; (4) confirmed by MRI or CT as a stroke; (5) unlimited duration; (6) not accompanied by severe cognitive impairment, aphasia, and difficulty in understanding; and (7) no serious heart, lung, liver, and kidney insufficiency and mental illness.

\subsection{Equipment and Methods}

2.2.1. DWI Method. Using GE's SignaHDe1.5T superconducting MR equipment, 8-channel head, and neck combined coil, all patients underwent cross-sectional DWI examination of 4 parameters and obtained 4 sets of images, using a single excitation plane echo sequence, and applied diffusion-sensitive gradients, diffusion gradient factors $(b$ value) in slice selection, phase encoding, and frequency encoding directions, respectively. The scanning schemes and parameters of each group are shown in Table 1, and scanning speed of each group is $1000 \mathrm{~s} / \mathrm{mm}^{2}$.

2.2.2. Sports Relearning Method. The patients were randomly divided into treatment group and control group. The control group used conventional Bobath treatment technology (neurodevelopmental therapy), including exercise treatment for the affected hand and wrist, control key points, reflex suppression, and induced normal posture reflex; the treatment group was treated with exercise relearning method. The training time of the two groups is basically the same: $1.0 \mathrm{~h}$ daily training, $5 \mathrm{~d}$ weekly training, and a total course of 12 weeks. The treatment group uses MRP, including (1) analysis of upper limb function: analysis of the affected hand, wrist, and arm; (2) and (3) exercise loss: triggering arm extension and finger muscle activity and movement control and triggering muscle activity and exercise control of training operations, training wrist extension, training arm rotation, training thumb abduction and rotation (to palm), training hand radial and ulnar side (opposed) and adduction abduction, and training to operate objects; let go of your fingers; and (4) transfer training to daily life. Each movement can range from passive movement to auxiliary movement and finally to active movement. The training is carried out by rehabilitation therapists who are trained in two treatment methods. Avoid pathological postures during training and develop normal exercise patterns for 60 minutes, once a day and 5 times a week. Assign homework to patients, and ask family members to assist in training. Check the completion of the work at the beginning, and then start a new practice project [2].

2.3. Evaluation of Image Quality. Two doctors with more than 8 years of experience in MR imaging diagnosis will evaluate the quality of the four groups of imaging. When the opinions are not consistent, the third chief physician will decide. According to the presence or absence of artifacts, noise size, and anatomical details, image scores are scored as follows: (1) poor image quality and cannot be diagnosed; (2) poor image quality and affects diagnosis; (3) good image quality and does not affect diagnosis; and (4) good image quality, suitable for diagnosis. The DWI image quality score is the sum of the scores of all levels divided by the number of layers [3].

2.4. Statistical Analysis. The comparison between multiple groups uses the Kruskal-Wallis $\mathrm{H}$ test, and the comparison between the two groups uses the Mann-Whitney $U$ test. $P<0.05$ is considered statistically significant. The mutual information of the two images is expressed as

$$
I(F, R)=H(F)+H(R)-H(F, R) .
$$

Among them,

$$
\begin{aligned}
H(F) & =-\sum_{f} p_{F}(f) \log _{2} p_{F}(f), \\
H(F, R) & =-\sum_{f r} p_{F, R}(f, r) \log _{2} p_{F, R}(f, r) .
\end{aligned}
$$

The normalized mutual information is expressed as

$$
\operatorname{NMI}(F, R)=\frac{H(F)+H(R)}{H(F, R)} .
$$

It is less affected by the size of the overlapping area of the image to be registered and has stronger robustness. In this paper, NMI is used as the objective function of registration. The process of image registration based on mutual information can be described as follows: for the $p$ point of the pixel value $f$ in $\mathrm{F}$, seek a geometric transformation $T_{\alpha}$ with the parameter $\alpha$, and map the $p$ point to $T_{\alpha}(p)$ on the $R$ pixel value $r$. The normalized mutual information $\operatorname{NMI}(F, R)$ of the two random variables $f=F(p)$ and $r=R\left(T_{\alpha}(p)\right)$ is maximized. That is,

$$
\alpha^{*}=\arg \max _{\alpha} \operatorname{NMI}(F, R)
$$

where $\alpha$ is the parameter vector of the geometric transformation $T$. Equation (4) shows that the realization process of the image registration method based on mutual information is a multiparameter optimization process. The optimized registration algorithm has an obvious flaw. When the point overlaps with the neighborhood point, that is, 
TABLE 1: Scanning parameters of 4 groups of DWI images.

\begin{tabular}{lcccccccc}
\hline Grouping & $\begin{array}{c}\text { TR } \\
(\mathrm{ms})\end{array}$ & $\begin{array}{c}\text { TE } \\
(\mathrm{ms})\end{array}$ & $\begin{array}{c}\text { Matrix } \\
(\mathrm{mm})\end{array}$ & $\begin{array}{c}\text { Number of incentives } \\
(\text { times })\end{array}$ & $\begin{array}{c}\text { FOV } \\
(\mathrm{cm})\end{array}$ & $\begin{array}{c}\text { Layer thickness/layer } \\
\text { spacing }(\mathrm{mm})\end{array}$ & $\begin{array}{c}\text { Parallel acquisition } \\
\text { technology }\end{array}$ & $\begin{array}{c}\text { Scan time } \\
(\mathrm{s})\end{array}$ \\
\hline 1 & 4777 & 110 & $96 \times 128$ & 4 & $25 \times 25$ & $5 / 1.5$ & No & 177 \\
2 & 4777 & 110 & $96 \times 128$ & 4 & $25 \times 25$ & $5 / 1.5$ & Have & 81 \\
3 & 4650 & 109 & $96 \times 128$ & 1 & $25 \times 25$ & $5 / 1.5$ & Have \\
4 & 3575 & 86.8 & $96 \times 128$ & 1 & $40 \times 40$ & $5 / 1.5$ & Have \\
\hline
\end{tabular}

when the two images to be registered have integer displacement on the coordinate axis, the four gray histograms originally involved in the calculation of the point suddenly change into one point. This causes a sudden change in entropy and the local maximum of the mutual information function. The local extremum formed by the optimized registration algorithm exists at every integer multiple displacement of the pixel, which will seriously interfere with the optimization of the mutual information function. If the extreme value at the integer multiple displacement of the pixel can be eliminated or reduced, then we find that the optimization process can become more stable. The reason why the optimal registration algorithm produces local extrema lies in the mutation of the joint distribution entropy. As long as this mutation is reduced, the local extremum can be reduced. Can we find a better coefficient allocation method so that the new coefficient should still ensure that the closer the point $n_{i}$ is to the point $s_{r}, w_{i}$, the larger; and $w_{i}$ is nonnegative, $\sum w_{i}\left(s_{r}\right)=1$. The B-spline basis function often used in graphics just meets such a condition. The B-spline basis function is expressed as

$$
N_{i, o}(u)=\frac{u-t_{i}}{t_{i+k-1}-t_{i}} N_{i, k-1}(u)+\frac{t_{i+k}-u}{t_{i+k}-t_{i+1}} N_{i+1, k-1}(u) .
$$

\section{Results}

3.1. Comparison of Image Quality Scores of 4 Groups. Group 2 had the highest image quality score, but there was no statistically significant difference with group $1(P>0.05)$. The score of group 3 is lower than that of groups 1 and 2 (both $P<0.001$ ), but higher than 3 , which does not affect the diagnosis. Group 4 score is $<3$ points and lower than the other 3 groups (all $P<0.001$ ), affecting the diagnosis. See Table 2 for details [4].

3.2. Diagnostic Results. Among the 50 patients with AIS, 4 had extensive cerebral infarction, 19 had multiple cerebral infarction (Figures 1 and 2), 14 had watershed cerebral infarction, and 13 had lacunar infarction [5].

Image A (group 1) has good image quality, with a score of 3.68 and a scanning time of 2 min $57 \mathrm{~s}$. Picture B (group 2) has the highest image quality, with clear boundaries between gray and white matter, with a score of 3.84 and a scan time of $1 \mathrm{~min}$ and $21 \mathrm{~s}$. Picture C (group 3 ) is more noisy, but the lesion can still be clearly displayed without affecting the diagnosis, with a score of 3.16 and a scan time of $23 \mathrm{~s}$. Panel D (group 4) failed to distinguish between gray and white matter, the boundary between brain tissue and lesions was blurred, artifacts (white arrows) appeared, the score was 2 points, and the scan time was $18 \mathrm{~s}$.

The ADC values of the A-D chart are $0.495 \times 10^{-3}$, $0.524 \times 10^{-3}, \quad 0.561 \times 10^{-3}$, and $0.538 \times 10^{-} 3 \mathrm{~mm}^{2} / \mathrm{s}$ in sequence.

3.3. Relationship between the Two Groups of Patients before and after Treatment. After treatment of 33 stroke patients in the treatment group with exercise relearning method for 12 weeks, the average increase in FMA was $8.64 \pm 3.86$ points, and 32 patients in the control group received conventional rehabilitation treatment for 12 weeks. After that, the FMA increased by an average of $6.22 \pm 2.84$ points. After treatment, the MBI of the two groups was improved compared with that before the treatment. The treatment group increased $37.27 \pm 18.03$ points and the control group increased $28.13 \pm 15.28$ points. There was also a statistically significant difference between the two groups $(P<0.05)$. It shows that the improvement of hand and wrist exercise ability of the treatment group is better than that of the control group, and the ability of daily life is also improved; see Tables 3 and 4.

\section{Discussion}

4.1. Scanning Technologyand b Value Selection. In this study, the SE-EPIDWI sequence was used. The small $b$ value of DWI reflected the microcirculation of local tissues to a certain extent, but the measured ADC value was less stable, susceptible to other physiological activities, and could not effectively reflect the diffusion of water molecules. DWI with large $b$ value is less affected by local tissue microcirculation perfusion and can better reflect the diffusion movement of water molecules [6]. However, as the $b$ value increases, the tissue signal attenuation becomes more obvious, and the image SI and SNR will decrease. Chen et al. found that the image quality obtained by brain DWI examination when $b=800-1000 \mathrm{~s} / \mathrm{mm}^{2}$ is good and the parameters are relatively stable, so the $b$ value selected in this study is $1000 \mathrm{~s} / \mathrm{mm}^{2}$.

4.2. Impact of Parallel Acquisition Technology on Images. Parallel acquisition technology uses the spatial position difference of multichannel surface coils to distinguish the source of the signal. Through the cooperation of each coil, the number of phase encoding steps in the imaging 
TABle 2: Details.

\begin{tabular}{lcccc}
\hline Group & SNR & CNR & SIR & Image quality score \\
\hline 1 & $402.31 \pm 272.00$ & $242.08 \pm 158.74$ & $2.60 \pm 0.45$ & $3.90(3.60 .4 .00)$ \\
2 & $473.83 \pm 458.93$ & $290.15 \pm 286.35$ & $2.65 \pm 0.54$ & $3.98(3.84 .4 .00)$ \\
3 & $388.80 \pm 324.57$ & $234.53 \pm 206.81$ & $2.56 \pm 0.48$ & $3.00(3.00 .3 .21)$ \\
4 & $559.61 \pm 467.60$ & $259.65 \pm 198.75$ & $2.04 \pm 0.46 \mathrm{abc}$ & $2.00(1.02 .2 .00)$ \\
$\chi^{2}$ & 2.021 & 0.645 & 17.444 & 150.799 \\
$P$ & 0.112 & 0.587 & $<0.001$ & $<0.001$ \\
\hline
\end{tabular}

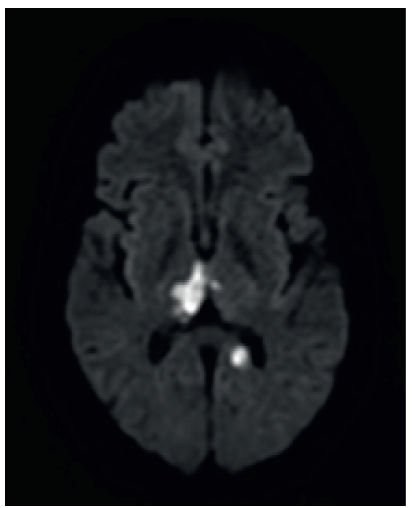

(a)

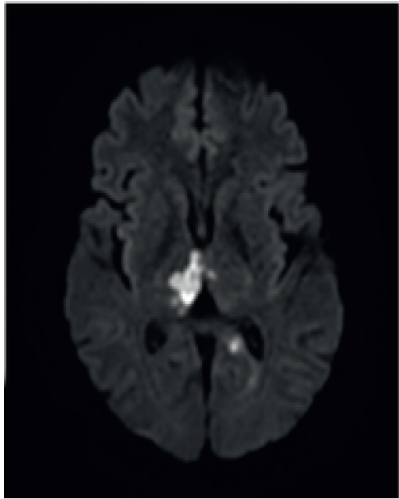

(b)

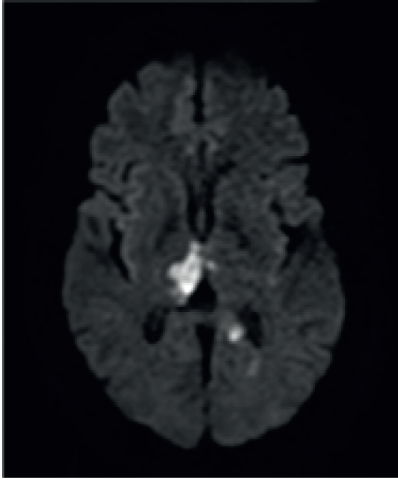

(c)

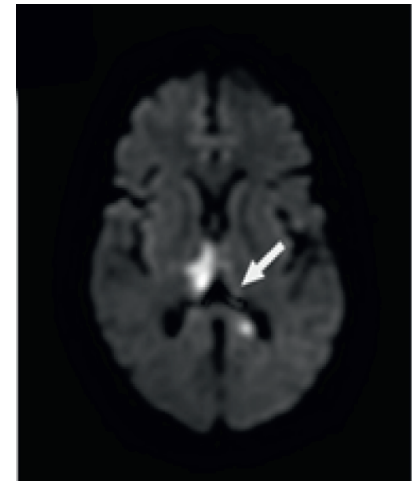

(d)

FIgURE 1: DWI images of 4 parameters of AIS patients.

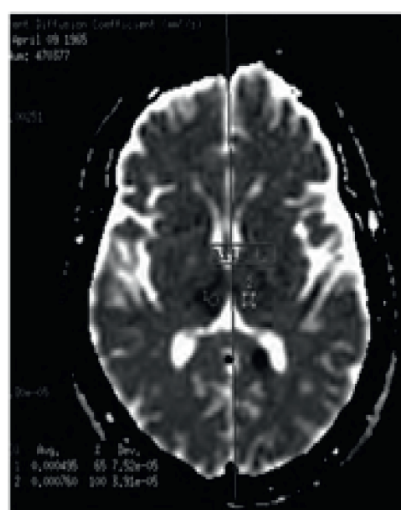

(a)

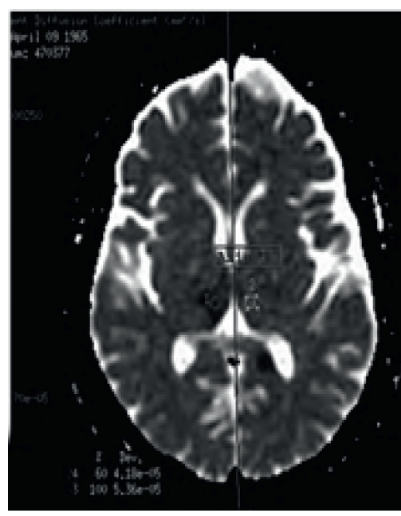

(b)

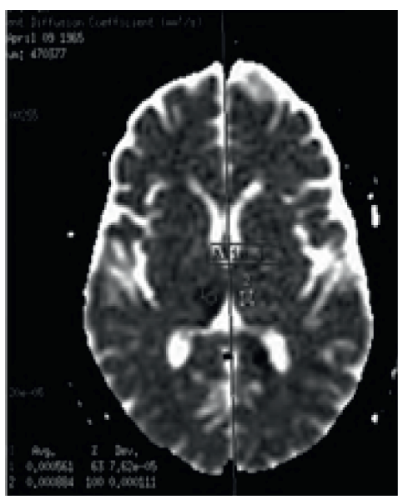

(c)

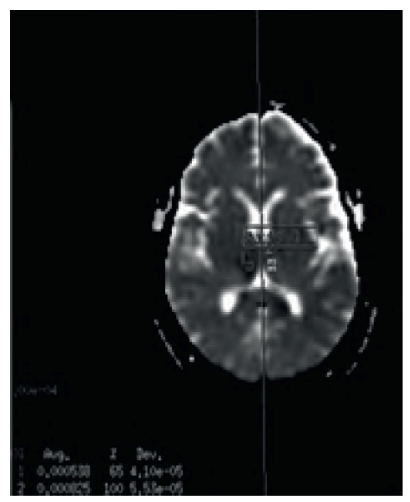

(d)

FIGURE 2: ADC diagram obtained by scanning 4 parameters of AIS patients.

TABLE 3: FMA analysis results of the treatment group and control group of stroke patients before and after treatment.

\begin{tabular}{lcccc}
\hline Group & $n$ & $\begin{array}{c}\text { Before } \\
\text { treatment }\end{array}$ & $\begin{array}{c}\text { After } \\
\text { treatment }\end{array}$ & $\begin{array}{c}\text { FMA } \\
\text { difference }\end{array}$ \\
\hline Control group & 32 & $5.09 \pm 3.17$ & $11.31 \pm 3.95$ & $6.22 \pm 2.84$ \\
Therapy group & 33 & $5.67 \pm 6.24$ & $14.30 \pm 5.70$ & $8.64 \pm 3.86$ \\
\hline
\end{tabular}

process is reduced, and the scanning time is significantly reduced. The improvement of $\mathrm{K}$-space filling method and reconstruction algorithm can improve the spatial resolution and reduce the susceptibility artifact in the same scanning time [7]. In this study, the basic scanning
TABLE 4: Comparison of MBI before and after treatment in patients with stroke.

\begin{tabular}{lcccc}
\hline Group & $n$ & $\begin{array}{c}\text { Before } \\
\text { treatment }\end{array}$ & $\begin{array}{c}\text { After } \\
\text { treatment }\end{array}$ & $\begin{array}{c}\text { MBI } \\
\text { difference }\end{array}$ \\
\hline Control group & 32 & $36.09 \pm 20.39$ & $64.22 \pm 17.60$ & $28.13 \pm 15.28$ \\
Therapy group & 33 & $43.03 \pm 17.36$ & $80.30 \pm 18.66$ & $37.27 \pm 18.03$ \\
\hline
\end{tabular}

parameters of group 1 and group 2 are the same, group 2 uses parallel acquisition technology, the image quality is improved compared with group 1 , the subjective reading score is the highest, the image quality is the best, and the scanning time is reduced by $1 \mathrm{~min} 36 \mathrm{~s}$ compared with group 1 . 


\subsection{Reducing the Impact of the Number of Excitations (NEX)} on the Image. In this study, group 3 used parallel acquisition technology and reduced NEX to 1, the time was 2 min $34 \mathrm{~s}$ less than that of group 1, the image quality score was lower than that of group $1(P<0.05)$, and the differences between SIR, SNR, CNR, ADC values, and group 1 were all different. There was no statistical significance [8]. Theoretically, according to the formula "scan time $=\mathrm{TR} \times n \times \mathrm{NEX}$ (TR is the repetition time), $\mathrm{SNRN}=\mathrm{NEX} 2 \times \mathrm{SNR} 1$," under the condition that the remaining parameters remain unchanged, reducing NEX can shorten the imaging time, and SNR is only the square root of NEX proportional, so NEX has a limited impact on SNR among the factors that affect image quality. This study also found that when the remaining parameters were unchanged, reducing NEX to 1 had little effect on the SIR, SNR, CNR, and ADC values of the image. Although the gray-white boundary of the image in the subjective reading is slightly blurred compared with group 1 , the image quality is slightly reduced, but the image quality score is more than 3 points, which does not affect the diagnosis. The scan time of group 3 is only $23 \mathrm{~s}$, which can be used as a personalized scan parameter for AIS patients [9].

4.4. Increasing the Impact of FOV on Images. In this study, the FOV of group 4 increased to 40 , and the scanning time was reduced by 2 minutes and 39 seconds compared with group 1 . The difference in image quality score, SIR, and group 1 was statistically significant, and the difference in ADC value, SNR, CNR, and group 1 was not statistically significant. The reason is that the larger the FOV, the larger the voxel, resulting in a decrease in spatial resolution, at the same time, the background noise of the image is reduced, and the SNR of the image is increased. Therefore, the SIR and image quality of group 4 decreased compared with group 1, but the SNR and CNR did not change significantly. Among the 4 groups of DWI images, the scan time of group 4 is the shortest, but the image quality is the worst, which cannot meet the diagnostic requirements [10].

4.5. Effect of Changing Parameters on ADC Value. Because the DWI signal strength is affected by the T2 penetration effect of the tissue, sometimes it does not truly reflect the speed of the diffusion of water molecules in living tissue. The quantitative index is by measuring the $\mathrm{ADC}$ value of the lesion. Therefore, this study measures the ADC value of each group to understand whether the ADC value will be changed after changing the parameters, thereby affecting the accuracy of the measured value. The ADC value is mainly calculated based on the SI change on the DWI image. In theory, the ADC value is mainly affected by the field strength, $b$ value, the spatial distribution of the imaging substance, and its internal molecules, but the reduced SNR of the image and the magnetic sensitivity artifacts will affect the ADC value measurement. The differences in the ADC values of the four groups of images in this study were not statistically significant, indicating that the adoption of parallel acquisition technology and the appropriate changes in NEX, TR, TE, and FOV parameters did not affect the accuracy of the ADC values measured [11].

4.6. Effect of Exercise Relearning on Patients' Memory Function. MRP is a kind of exercise therapy proposed by Australian scholars Carr and Shepherd in the early 1980s. It regards the recovery training of motor function after central nervous system injury as a process of relearning and retraining. Mainly based on the theoretical basis of neurophysiology, sports science, biomechanics, behavioral science, and so on and oriented by work or function, under the premise of emphasizing active participation of patients and no serious cognitive impairment, reeducate patients according to scientific sports learning methods to restore its motor function. Subsequently, the two scholars proposed training guidelines for training key actions and optimizing motor skills for stroke patients with hemiplegia based on the theory and scientific research results of brain plasticity. Emphasize the organization of the environment and training, encourage patients to actively participate in active training and learning, and optimize exercise skills. MRP functional retraining is an important condition for central nervous system functional reorganization. In a randomized controlled study, a group of patients used exercise to relearn the functional intensive training of their opponents and wrists. After proton emission imaging, they found brain changes and deduced that training induced functional reorganization of the bilateral motor and sensory systems of the brain. The study found that exercise relearning can not only promote the recovery of motor function in patients with stroke but also shorten the length of hospital stay and improve the patients' ability of daily life. The study found that, in stroke patients, the use of MRP is better than the use of Bobath technology to improve the recovery of motor function. A randomized controlled study found that motor relearning can promote the recovery of motor function of the paralyzed hand and wrist of stroke patients. Scholars at home and abroad are studying the mechanism of motor function recovery after brain injury. The results show that the theory of ipsilateral brain domination, nerve regeneration, and brain plasticity and brain function reorganization have laid a solid theoretical foundation for the development of neurorehabilitation medicine [12].

The treatment group in this study adopted MRP treatment, and the rehabilitation of the upper limb hand and wrist of the hemiplegic side no longer stopped to produce the correct movement but paid more attention to the use function in daily life, that is, the quality function. In the treatment, the treatment plan is individualized, which varies according to the condition and has some emphasis. At the right time, pay attention to the patient's tolerance and adjust the amount of activity, and pay attention to the complexity of the use of the hand and wrist and perform some functional activities and so on independently, emphasizing repeated training, meaningful task-oriented exercises, intensive use of affected limbs, two-handed exercises, and so on, step by step. The results of this study show that MRP can not only improve the motor function of the upper limb hand 
and wrist of stroke patients but also improve the daily living activities of patients. It is suggested that the improvement of hand and wrist exercise ability and daily activity ability of MRP is better than conventional Bobath treatment. In the future, it is necessary to conduct a larger sample of research on cases of stroke for half a year, combined with the study of functional magnetic resonance imaging to understand the changes in neurological imaging of MRP on upper limb hand and wrist functional rehabilitation in patients with hemiplegia [13].

\section{Conclusion}

In neurorehabilitation treatment, motor function training and daily life skills are closely combined. It varies from person to person and from illness to condition. Combined with the patient's mental state, repeated practice can lay the foundation for regaining the correct exercise mode. The patient is practicing. Among them, certain task-oriented activities should be taken, and training for daily life activities and the use of sports relearning therapy can make stroke patients more effective.

\section{Data Availability}

The data used to support the findings of this study are available from the corresponding author upon request.

\section{Conflicts of Interest}

The authors declare no conflicts of interest.

\section{Acknowledgments}

This work was supported by the China Rehabilitation Hospital Association Fund (no. 20160209).

\section{References}

[1] G. M. Lutokhin, L. A. Geraskina, L. A. Geraskina, A. V. Fonyakin, and M. Y. Maksimova, "Optimization of early rehabilitation of patients with ischemic stroke and sleepdisordered breathing," Human Physiology, vol. 44, no. 8, pp. 875-882, 2018.

[2] Y. Xu, S. Wu, M. Wang, and Y. Zou, "Design and implementation of distributed RSA algorithm based on Hadoop," Journal of Ambient Intelligence and Humanized Computing, vol. 11, pp. 1047-1053, 2020.

[3] R. Kumar Sahu and V. V. Thakare, "Optimization of symmetric linear phase low pass fir filter using genetic algorithm," Acta Radiologica, vol. 45, no. 6, pp. 679-688, 2015.

[4] W. Chen, J. Zhang, J. Zhang, D. Long, Z. Wang, and J.-M. Zhu, "Optimization of intra-voxel incoherent motion measurement in diffusion-weighted imaging of breast cancer," Journal of Applied Clinical Medical Physics, vol. 18, no. 3, pp. 191-199, 2017.

[5] S. Wu, "A traffic motion object extraction algorithm," International Journal of Bifurcation and Chaos, vol. 25, no. 14, Article ID 1540039, 2015.

[6] H. Shalihah, A. Kusumaatmaja, A. Kusumaatmaja, A. D. Nugraheni, and K. Triyana, "Optimization of chitosan/ PVA concentration in fabricating nanofibers membrane and its prospect as air filtration," Materials Science Forum, vol. 901, pp. 20-25, 2017.

[7] X. Milidonis, I. Marshall, I. Marshall, M. R. Macleod, and E. S. Sena, "Magnetic resonance imaging in experimental stroke and comparison with histology," Stroke, vol. 46, no. 3, pp. 843-851, 2015.

[8] S. Wu, M. Wang, and Y. Zou, "Research on internet information mining based on agent algorithm," Future Generation Computer Systems, vol. 86, pp. 598-602, 2018.

[9] B.-S. Park, J. W. Noh, J.-W. Noh et al., "A comparative study of the effects of trunk exercise program in aquatic and landbased therapy on gait in hemiplegic stroke patients," Journal of Physical Therapy Science, vol. 28, no. 6, pp. 1904-1908, 2016.

[10] H. Y. Lee, Y. L. Kim, Y. L. Kim, and S. M. Lee, "Effects of virtual reality-based training and task-oriented training on balance performance in stroke patients," Journal of Physical Therapy Science, vol. 27, no. 6, pp. 1883-1888, 2015.

[11] N. N. Wei, J. X. Pan, Y. P. Chen, and Y. Chen, "Effects of balance acupuncture combined with motor relearning for lower limb motor function of stroke patients with hemiplegia," Randomized Controlled Trial, vol. 43, no. 11, pp. 730-732, 2018.

[12] Y. B. Il, S. B. Kyoung, and J. S. Mi, "Effects of two-handed task training on upper limb function of chronic hemiplegic patients after stroke," Journal of Physical Therapy Science, vol. 29, no. 1, pp. 102-105, 2017.

[13] J. X. Pan, Y. P. Chen, and N. N. Wei, "Improvement of upper limb and hand functions of stroke patients by balancing acupuncture combined with motor relearning training," Acupuncture Research, vol. 43, no. 2, pp. 123-126, 2018. 